\title{
Bayesian Estimation for the Birnbaum-Saunders Distribution in the Presence of Censored Data
}

\author{
F. A. Moala, J. A. Achcar and R. P. Gimenez
}

\begin{abstract}
The use of Birnbaum-Saunders distribution can be a good alternative for analyzing the equipment lifetime data. In this work two different prior distributions are used in the estimation of the parameters of the Birnbaum-Saunders distribution under the Bayesian approach and the presence of censored data type I and II. Assuming a prior dependence between the parameters, an alternative prior distribution based on copula function approach is proposed. Thus, a study to check if the priors lead to the same posterior inference is of great practical interest. Two examples are presented to illustrate the proposed methodology and investigate the performance of the prior distributions. The Bayesian analysis is performed based on the Markov Chain Monte Carlo (MCMC) method to generate the posterior distribution samples.
\end{abstract}

Keywords - Birnbaum-Saunders distribution, censorship Type I, Type II, copula, MCMC.

\section{INTRODUÇÃO}

$\mathrm{B}$ IRNBAUM-SAUNDERS [1-2] introduziram uma distribuição de probabilidade que pudesse ser derivada de um processo de repetidos ciclos de estresse físico que causasse danos em materiais levando assim à uma eventual falha.

A variável aleatória $T$ segue uma distribuição BirnbaumSaunders, denotada por $B S$, se sua densidade de probabilidade é dada por,

$f(t)=\frac{\left(t^{2}-\beta^{2}\right) \exp \left[-\frac{1}{2 \alpha^{2}}\left(\frac{t}{\beta}+\frac{\beta}{t}-2\right)\right]}{2 \sqrt{2 \pi \sqsubset \alpha \beta \mathrm{t}^{2}}\left[\left(\frac{t}{\beta}\right)^{\frac{1}{2}}-\left(\frac{\beta}{t}\right)^{\frac{1}{2}}\right]}$,

onde $t>0, \alpha>0$ e $\beta>0$.

Os parâmetros $\alpha$ e $\beta$ são, respectivamente, parâmetros de forma e escala. Nas Fig. 1, 2 e 3, temos os gráficos da densidade, função de sobrevivência e função de risco assumindo diferentes valores para $\alpha$. Observamos uma grande flexibilidade de ajuste da distribuição a dados de tempo de vida. A correspondente função de sobrevivência é dada por:

$S(t)=1-\Phi\left\{\frac{1}{\alpha}\left[\left(\frac{t}{\beta}\right)^{\frac{1}{2}}-\left(\frac{\beta}{t}\right)^{\frac{1}{2}}\right]\right\}$,

onde $\Phi(Z)$ é a função de distribuição acumulada normal padrão. A média e a variância de $T$ são, respectivamente, dadas por,

$E(T)=\beta\left(1+\frac{\alpha^{2}}{2}\right)$,

F. A. Moala, UNESP, Faculdade de Ciências e Tecnologia, Presidente Prudente,Brasil,femoala@fct.unesp.br

J. A. Achcar, USP, Faculdade de Medicina, Ribeirão Preto, Brasil, achcar@fmrp.usp.br

R. P. Gimenez, UNESP, Faculdade de Ciências e Tecnologia, Presidente Prudente, Brasil, robsongimenezz@hotmail.com e

$\operatorname{var}(T)=(\alpha \beta)^{2}\left(1+\frac{5 \alpha^{2}}{4}\right)$.

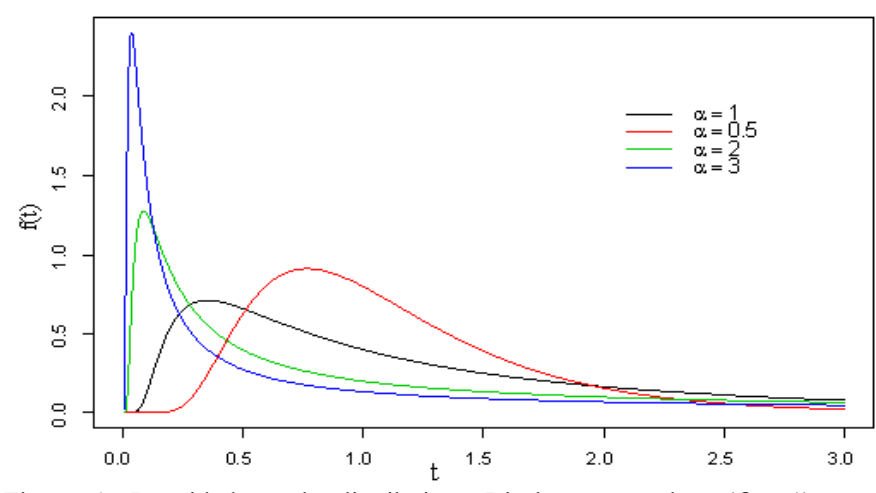

Figura 1. Densidades da distribuição Birnbaum-Saunders $(\beta=1)$ para diferentes valores de $\alpha$.

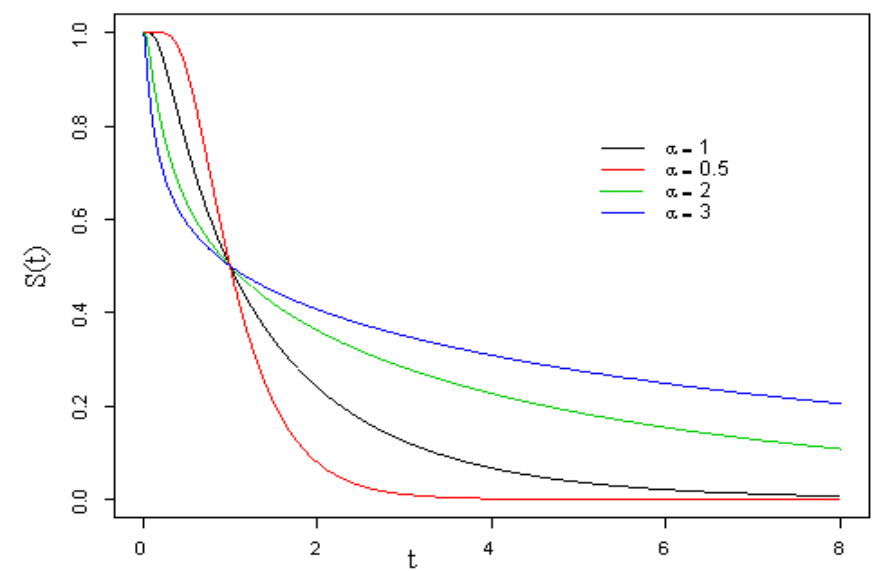

Figura 2. Funções de sobrevivência da distribuição Birnbaum-Saunders $(\beta=1)$ para diferentes valores de $\alpha$.

Amostras aleatórias provenientes da distribuição $B S$ podem ser geradas a partir de uma variável aleatória $Z$ sob distribuição normal padrão através da relação (veja [3]):

$T=\beta\left(1+\frac{\alpha^{2} Z^{2}}{2}+\alpha Z \sqrt{\frac{\alpha^{2} Z^{2}}{4}+1}\right)$.

A função de risco $h(t)=\frac{f(t)}{1-F(t)}$ (ver [4]) é determinada a partir de (1) e (2) por,

$h(t ; \alpha, \beta)=\frac{\left(t^{2}-\beta^{2}\right) \exp \left[-\frac{1}{2 \alpha^{2}}\left(\frac{t}{\beta}+\frac{\beta}{t}-2\right)\right]}{2 \sqrt{2 \pi} \alpha \beta t^{2}\left[\left(\frac{t}{\beta}\right)^{\frac{1}{2}}-\left(\frac{\beta}{t}\right)^{\frac{1}{2}}\right] 1-\Phi\left\{\frac{1}{\alpha}\left[\left(\frac{t}{\beta}\right)^{\frac{1}{2}}-\left(\frac{\beta}{t}\right)^{\frac{1}{2}}\right]\right\}}$. 
Uma análise Bayesiana para a distribuição BirnbaumSaunders com densidade (1), considerando densidades a priori não-informativas para os parâmetros $\alpha$ e $\beta$, é realizada nesse artigo.

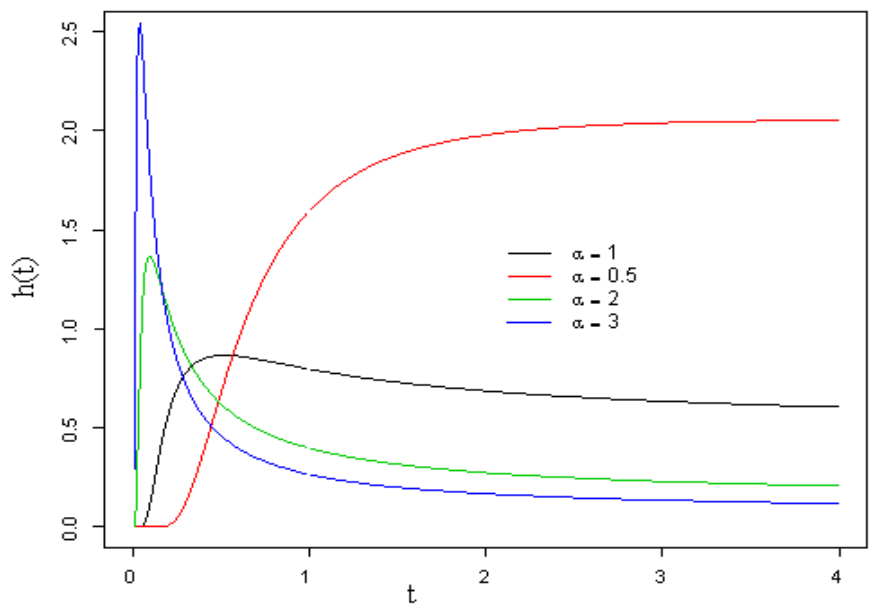

Figura 3. Funções de risco da distribuição Birnbaum-Saunders $(\beta=1)$ para diferentes valores de $\alpha$.

O trabalho está organizado da seguinte forma: na seção 2 apresentamos as funções de verossimilhança para dados sob censura Tipo I e II. Na seção 3 a análise Bayesiana para a distribuição Birnbaum-Saunders é proposta. $\mathrm{Na}$ seção 4, apresentamos exemplos de estimação para os casos de dados censurados tipo I e II e seus efeitos numa análise estatística. Finalmente na seção 5, apresentamos algumas conclusões.

\section{FUNÇÃO DE VEROSSIMILHANÇA SOB DADOS CENSURADOS}

Um esquema de censura do tipo I pode ser descrito como se segue.

Seja uma amostra aleatória $t_{1}, t_{2} \ldots, t_{n}$ de tamanho $n$ de tempos de vida sob distribuição com densidade $f(t)$. Na presença de dados censurados ou não-censurados definimos a variável indicadora como:

$\delta_{i}=\left\{\begin{array}{ll}1, & t_{i}=T_{i} \\ 0, & t_{i}=L_{i}\end{array}\right.$,

onde $t_{i}=\min \left(T_{i}, L_{i}\right)$ é o tempo observado, $T_{i}$ é o tempo de sobrevivência e $L_{i}$ é o tempo de censura assumido fixo para o i-ésimo indivduo. Neste caso, a função de verossimilhança para o parâmetro é dada (ver, por exemplo, [4]) por:

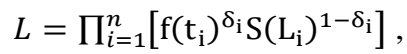

onde $S(t)$ é a função de sobrevivência.

Se os tempos de vida útil dos componentes em teste seguem a distribuição BS dada em (1) sob esquema de censura tipo I, então a função de verossimilhança para os parâmetros $\alpha e \beta$ pode ser escrita como:

$L(\boldsymbol{\alpha}, \boldsymbol{\beta} \mid \boldsymbol{t})=\frac{1}{\boldsymbol{\alpha}^{r}} \prod_{i=1}^{n}\left[\boldsymbol{g}_{2}\left(\frac{\boldsymbol{t}_{\boldsymbol{i}}}{\boldsymbol{\beta}}\right)\right]^{\boldsymbol{\delta}_{\boldsymbol{i}}}\left[1-\Phi\left(\frac{1}{\alpha} g_{1}\left(\frac{L_{i}}{\beta}\right)\right)\right]^{1-\delta_{i}} \times$ $\times \exp \left\{-\frac{1}{2 \alpha^{2}} \sum_{i=1}^{n} \delta_{i} g_{3}\left(\frac{t_{i}}{\beta}\right)\right\}$,

com funções definidas como $g_{1}(t)=\sqrt{t}-\frac{1}{\sqrt{t}}, g_{2}(t)=\sqrt{t}+$ $\frac{1}{\sqrt{t}}$ e $g_{3}(t)=t+\frac{1}{t}-2$,

Num esquema de censuras tipo II, colocamos $n$ unidades em teste e terminamos o experimento após a ocorrência da résima falha ( $r$ fixo, $0 \leq r \leq n$ ).

A função de verossimilhança baseada nos $r$ tempos de falhas $t_{(1)}, t_{(2)} \ldots, t_{(r)}$ é dada por

$L=\frac{n !}{(n-r) !} f\left(t_{(1)}\right) f\left(t_{(2)}\right) \ldots f\left(t_{(r)}\right)$.

Deste modo, para os tempos de falha dos componentes ajustados a uma distribuição $B S$ dada em (1), a função de verossimilhança correspondente ao esquema de censura tipo II é dada por:

$$
\begin{aligned}
& L(\boldsymbol{\alpha}, \boldsymbol{\beta} \mid \boldsymbol{t})=\frac{1}{\alpha^{r}} \prod_{i=1}^{r}\left[g_{2}\left(\frac{t_{(i)}}{\beta}\right)\right]\left[1-\Phi\left(\frac{1}{\alpha} g_{1}\left(\frac{t_{(r)}}{\beta}\right)\right)\right]^{n-r} \times \\
& \times \exp \left\{-\frac{1}{2 \alpha^{2}} \sum_{i=1}^{r} g_{3}\left(\frac{t_{(i)}}{\beta}\right)\right\} .
\end{aligned}
$$

\section{ESTIMAÇÃO BAYESIANA}

Para uma análise Bayesiana do esquema de dados censurados, podemos considerar diferentes distribuições a priori para os parâmetros $\alpha$ e $\beta$.

Uma escolha natural para as distribuições a priori de $\alpha e \beta$ seria assumir que os dois parâmetros são independentes com distribuição Gama com as seguintes formas;

$\pi_{\alpha}(\alpha) \sim \operatorname{Gama}\left(a_{\alpha}, b_{\alpha}\right)$ e $\pi_{\beta}(\beta) \sim \operatorname{Gama}\left(a_{\beta}, b_{\beta}\right)$,

onde $a_{\alpha}, b_{\alpha}, a_{\beta}$ e $b_{\beta}$ são hiperparâmetros conhecidos e $\operatorname{Gama}(\mathrm{a}, \mathrm{b})$ indica uma distribuição gama, com uma média $\frac{a}{b} \mathrm{e}$ variância $\frac{a}{b^{2}}$. Os hiperparâmetros em (12) podem ser escolhidos como 0,01 ou 0,001 para representar ausência de informação a priori.

Agora, assumindo dependência entre os parâmetros $\alpha e \beta$, poderíamos também considerar uma distribuição bivariada a priori derivada de funções cópula (ver, por exemplo, [5], ou [6-7]).

Um caso especial é dado pela Copula Farlie-GumbelMorgenstern (ver [8]) com correspondente densidade conjunta a priori para $\alpha$ e $\beta$ condicionado a $\rho$, dada por

$$
\pi(\alpha, \beta \mid \rho)=f_{1}(\alpha) f_{2}(\beta)+\rho f_{1}(\alpha) f_{2}(\beta)\left[1-2 F_{1}(\alpha)\right]\left[1-2 F_{2}(\beta)\right],
$$

onde $f_{1}(\alpha)$ e $f_{2}(\beta)$ são as densidades marginais das quantidades aleatórias $\alpha e \beta, \quad F_{1}(\alpha) e F_{2}(\beta)$ são as correspondentes funções de distribuição marginais para $\alpha e \beta$. Observe que se $\rho=0$ temos independência entre $\alpha$ e $\beta$.

Diferentes escolhas podem ser consideradas como distribuições marginais para $\alpha$ e $\beta$ como as distribuições gama, exponencial, Weibull ou uniforme. 
Neste artigo, vamos supor distribuição marginal exponencial para $\alpha$ e $\beta$ com médias $a_{1}$ e $a_{2}$, respectivamente. Isto é,

$$
\pi(\alpha, \beta \mid \rho)=\exp \left\{-\frac{\alpha}{a_{1}}-\frac{\beta}{a_{2}}\right\}\left[1+\rho\left(2 \mathrm{e}^{-\frac{\alpha}{a_{1}}}-1\right)\left(2 \mathrm{e}^{-\frac{\beta}{a_{2}}}-1\right)\right]
$$

onde $\alpha>0$ e $\beta>0$.

Em geral, muitas diferentes prioris podem ser utilizados para $\rho$, uma possibilidade é a de considerar

$\pi(\rho) \propto\left(1-\rho^{2}\right)^{c}$,

com um valor especificado para a constante $c$; outra possibilidade é a de considerar uma distribuição a priori uniforme para $\rho$ sobre o intervalo [-1. 1].

A distribuição conjunta a posteriori para os parâmetros $\alpha$ e $\beta$ é proporcional ao produto das funções de verossimilhanças (9) e (11) e a priori $\pi(\alpha, \beta \mid \rho)$, conforme o tipo de censura presente dos dados.

Sumários a posteriori de interesse podem ser obtidos utilizando os métodos MCMC (Markov Chain Monte Carlo), como o popular algoritmo Amostrador de Gibbs [9], ou o algoritmo Metropolis-Hastings [10].

Desta forma, simulamos amostras para a distribuição conjunta a posteriori para $\alpha, \beta$ e $\rho$ das distributições conditionais $p(\alpha \mid \beta, \rho, \boldsymbol{t}), p(\beta \mid \alpha, \rho, \boldsymbol{t})$ e $p(\rho \mid \alpha, \beta, \boldsymbol{t})$ onde $\boldsymbol{t}$ é o vetor de dados.

\section{RESULTADOS}

Nesta seção, apresentamos aplicações práticas dos resultados teóricos discutidos nas seções anteriores com dois exemplos. O primeiro exemplo envolve dados sob censura Tipo I e o segundo censura tipo II.

\section{A. Presença de censura tipo I}

Considere uma situação em que peças de equipamentos são instaladas em diferentes instantes. Depois de algum tempo, algumas das peças falharão e as restantes ainda estarão funcionando. Os resultados deste experimento para 20 peças do equipamento são dados abaixo:

\section{$613193249^{+} 8^{+} 13^{+} 254150915274553^{+} 1218^{+} 3246^{+}$ $60^{+}$}

O sinal + indica que o dado foi censurado.

Assumimos a distribuição Birnbaum-Saunders com densidade (1) e consideramos as distribuições a priori dadas em (12) e (14) para o vetor de parâmetros $(\alpha, \beta)$. Usando o auxílo do software $\mathrm{R}$, temos nas tabelas I, II e III, os estimadores a posteriori de interesse com base em uma cadeia MCMC gerada de tamanho 15000, após um "burn-in" de tamanho 5000. A convergência do algoritmo foi monitorada através dos plots temporais e de autocorrelação para as amostras geradas e sugeriram convergência das cadeias. Os estimadores pontuais com respectivos desvios-padrão e os correspondentes intervalos de confiança para os parâmetros $\alpha$ e $\beta$ são apresentados nas tabelas I e II.
A tabela III a seguir fornece os estimadores e intervalos de confiança para os momentos (3) e (4) da distribuição $B S$.

TABELA I. SUMÁRIOS E INTERVALOS PARA $\alpha$.

\begin{tabular}{|c|c|c|c|}
\hline Método & Estimador & DP & IC 95\% \\
\hline prioris Gama & 0.88 & 0.19 & $(0.59,1.34)$ \\
\hline priori Cópula & 0.94 & 0.22 & $(0.61,1.46)$ \\
\hline
\end{tabular}

TABELA II. SUMÁRIOS E INTERVALOS PARA $\beta$.

\begin{tabular}{|c|c|c|c|}
\hline Método & Estimador & DP & IC 95\% \\
\hline prioris Gama & 29.43 & 6.60 & $(19.66,45.42)$ \\
\hline priori Cópula & 31.88 & 8.26 & $(20.18,51.87)$ \\
\hline
\end{tabular}

TABELA III. ESTIMADORES E INTERVALOS PARA A MÉDIA E VARIÂNCIA DA $B S$.

\begin{tabular}{|c|c|c|c|c|}
\hline Método & $\mathrm{E}(\mathrm{T})$ & $\mathrm{IC} \mathrm{95 \%}$ & $\mathrm{DP}(\mathrm{T})$ & $\mathrm{IC} \mathrm{95 \%}$ \\
\hline $\begin{array}{c}\text { prioris } \\
\text { Gama }\end{array}$ & 41.86 & $(25.97,77.53)$ & 38.58 & $(17.67,93.55)$ \\
\hline $\begin{array}{c}\text { priori } \\
\text { Cópula }\end{array}$ & 47.63 & $(27.53,96.63)$ & 47.11 & $(19.24,125.54)$ \\
\hline
\end{tabular}

Para uma comparação visual das prioris propostas neste artigo, a Fig. 4 mostra as posterioris marginais para $\alpha$ e $\beta$.
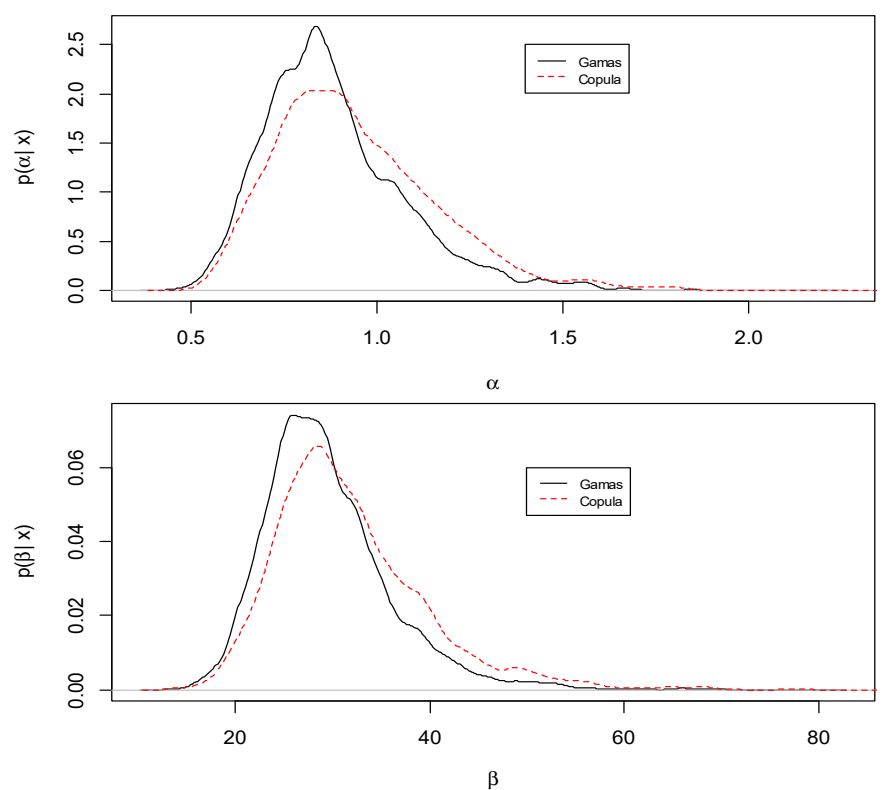

Figura 4. Plots das posterioris marginais para os parâmetros da BirnbaumSaunders.

Comparando as densidades marginais na Fig. 4, podemos ver que as posterioris correspondentes às prioris gamas são um pouco mais concentrada em torno de seu centro, implicando uma menor variância do que à fornecida pela priori cópula. Este comportamento em ambos os parâmetros pode também ser evidenciado, quando observa-se os sumários nas tabelas I, II e III.

O objetivo agora é obter o estimador a posteriori da função de sobrevivência $S(t)$ dada em (2). Isto é feito determinando-se 
$S(t) \mid \boldsymbol{t}=\iint P\{T>t \mid \alpha, \beta\} p(\alpha, \beta \mid \boldsymbol{t}) d \alpha d \beta$,

onde $p(\alpha, \beta \mid \boldsymbol{t})$ é a densidade a posteriori conjunta de $\alpha$ e $\beta$.

$\mathrm{Na}$ Fig. 5 estão representadas as funções de sobrevivência estimadas para cada um dos conjuntos de prioris (gamas e cópula). Pode-se observar nesta figura que a probabilidade de sobrevivência estimada sob a priori cópula será maior que a probabilidade estimada sob a priori gama ao longo do tempo. Neste caso, a estimação da função de sobrevivência é muito sensível à escolha da priori, ao considerarmos os dados sob censura tipo I neste exemplo.

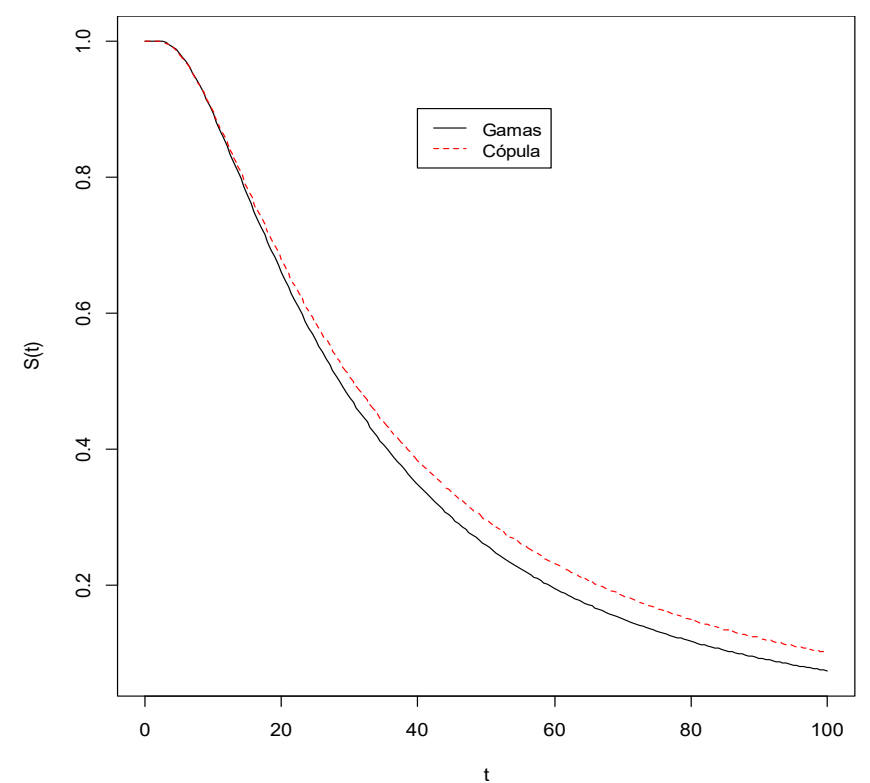

Figura 5. A função de sobrevivência estimada para prioris Gamas e cópula do Exemplo 1.

\section{B. Presença de censura tipo II}

[11] considera um experimento sobre o tempo de vida em horas de um certo tipo de rolamento. 10 rolamentos são testados e seus tempos de vida são apresentados na tabela IV. Neste caso, temos uma amostra censurada Tipo-II de tamanho $n=8$. Trata-se de uma base de dados muito pequena com 10 observações.

TABELA IV. DADOS DE TEMPO DE FADIGA APRESENTADOS POR MCCOOL (1974).

\begin{tabular}{|l|l|l|l|l|l|l|l|l|l|}
\hline 152 & 172 & 172 & 173 & 193 & 204 & 216 & 234 & 262 & 422 \\
\hline
\end{tabular}

Os estimadores pontuais com respectivos desvios-padrão e os correspondentes intervalos de confiança para os parâmetros $\alpha$ e $\beta$ são apresentados nas tabelas V e VI.

TABELA V. SUMÁRIOS E INTERVALOS DE CONFIANÇA PARA $\alpha$.

\begin{tabular}{|c|c|c|c|}
\hline Método & Estimador & DP & IC 95\% \\
\hline prioris Gama & 0.21 & 0.07 & $(0.12 ; 0.40)$ \\
\hline priori Cópula & 0.24 & 0.09 & $(0.13 ; 0.47$ \\
\hline
\end{tabular}

TABELA VI. SUMÁRIOS E INTERVALOS DE CONFIANÇA PARA $\beta$.

\begin{tabular}{|c|l|c|c|}
\hline Método & Estimador & DP & IC 95\% \\
\hline prioris Gama & 199.71 & 14.24 & $(172.90 ; 229.09)$ \\
\hline priori Cópula & 202.52 & 17.75 & $(173.01 ; 240.41)$ \\
\hline
\end{tabular}

Examinando os resultados nas tabelas V e VI observamos não haver grande diferença entre os estimadores e intervalos provenientes das duas prioris em estudo.

TABELA VII. ESTIMADORES E INTERVALOS PARA A MÉDIA E VARIÂNCIA DA $B S$.

\begin{tabular}{|c|c|c|l|c|}
\hline Método & $\mathrm{E}(\mathrm{T})$ & \multicolumn{1}{|c|}{$\mathrm{IC} 95 \%$} & $\mathrm{DP}(\mathrm{T})$ & $\mathrm{IC} 95 \%$ \\
\hline prioris & 204.96 & $(179.00,239.54)$ & 45.17 & $(24.63,90.715)$ \\
Gama & $(15.44)$ & & $(17.40)$ & \\
\hline priori & 209.47 & $179.41(257.11)$ & 52.22 & $(26.563,113.38)$ \\
Cópula & $(21.92)$ & & $(27.48)$ & \\
\hline
\end{tabular}

A Fig. 6 a seguir mostra realmente que as posterioris marginais para $\alpha$ e $\beta$ são bastante similares para as prioris gama e cópula. Portanto, neste caso, a escolha das prioris torna-se irrelevante.
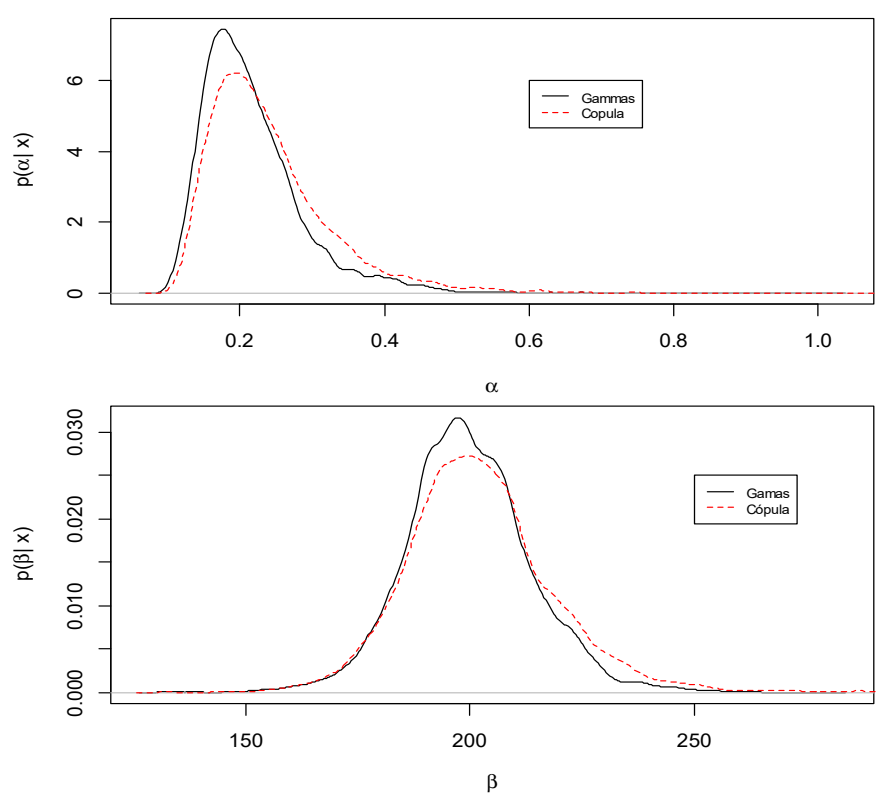

Figura 6. Plots das posterioris marginais para os parâmetros da Birbaum Saunders.

A Fig. 7 mostra graficamente as probabilidades de sobrevivência sob as duas prioris analisadas. Não há diferença entre as probabilidades de sobrevivência.

\section{Comparação do efeito da censura na estimação}

O fato de não se considerar os dados censurados em análises estatísticas pode provocar severa distorção das inferências. Assim, nesta seção examinamos o efeito de levarmos em conta a censura baseando-se nos dados dos exemplos 1 e 2 . 


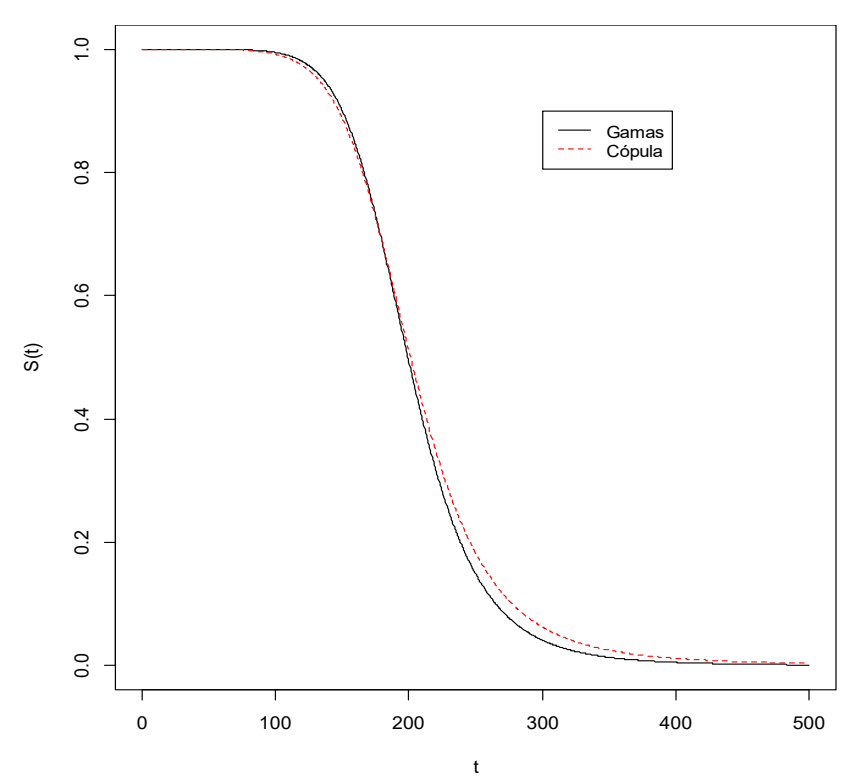

Figura 7. A função de sobrevivência estimada para prioris Gamas e cópula do Exemplo 2.

Primeiramente, considere os dados de censura tipo I do Exemplo 1. A priori utilizada aqui foi a priori (12) obtida como produto de gamas.

As tabelas VIII e IX mostram estimadores pontuais e intervalos de confiança a fim de uma comparação das inferências obtidas através de dados censurados e completos (não-censurados).

TABELA VIII. SUMÁRIOS E INTERVALOS DE CONFIANÇA PARA $\alpha$.

\begin{tabular}{|c|c|c|c|}
\hline Método & Estimador & D.P. & I.C. 95\% \\
\hline censurado & 0.88 & 0.19 & $(0.59,1.34)$ \\
\hline completo & 0.76 & 0.13 & $(0.55,1.06)$ \\
\hline
\end{tabular}

TABELA IX. SUMÁRIOS E INTERVALOS DE CONFIANÇA PARA $\beta$.

\begin{tabular}{|c|c|c|c|}
\hline Método & Estimador & D.P. & I.C. 95\% \\
\hline censurado & 29.43 & 6.60 & $(19.66,45.42)$ \\
\hline completo & 22.94 & 3.74 & $(16.37,31.36)$ \\
\hline
\end{tabular}

Os efeitos da utilização dos dados censurados numa análise estatística também podem ser verificados por meio gráfico. A Fig. 8 apresenta as funções de sobrevivência considerando os dados não-censurados e na presença de censura tipo I.

Neste gráfico fica evidente o efeito de não considerarmos os dados censurados na análise. Pode-se observar que a curva de sobrevivência para o caso sem censura fica sempre abaixo da correspondente aos dados censurados. Isto significa que a probabilidade de vida de uma determinada peça de equipamento seria sub-estimada se não considerássemos os valores censurados.

Considere agora a análise dos dados do Exemplo 2 supondo não haver a presença de censura e considerando a censura do tipo II. Em ambos os casos utilizamos a priori cópula dada em (14).

TABELA X. SUMÁRIOS E INTERVALOS DE CONFIANÇA PARA $\alpha$.

\begin{tabular}{|c|c|c|c|}
\hline Método & Estimador & DP & IC 95\% \\
\hline censurado & 0.24 & 0.09 & $(0.13 ; 0.47)$ \\
\hline completo & 0.10 & 0.35 & $(0.21 ; 0.61)$ \\
\hline
\end{tabular}

TABELA XI. SUMÁRIOS E INTERVALOS DE CONFIANÇA PARA $\beta$.

\begin{tabular}{|c|c|c|c|}
\hline Método & Estimador & DP & IC 95\% \\
\hline censurado & 202.52 & 17.75 & $(173.01 ; 240.41)$ \\
\hline completo & 23.98 & 210.07 & $(163.77 ; 259.19)$ \\
\hline
\end{tabular}

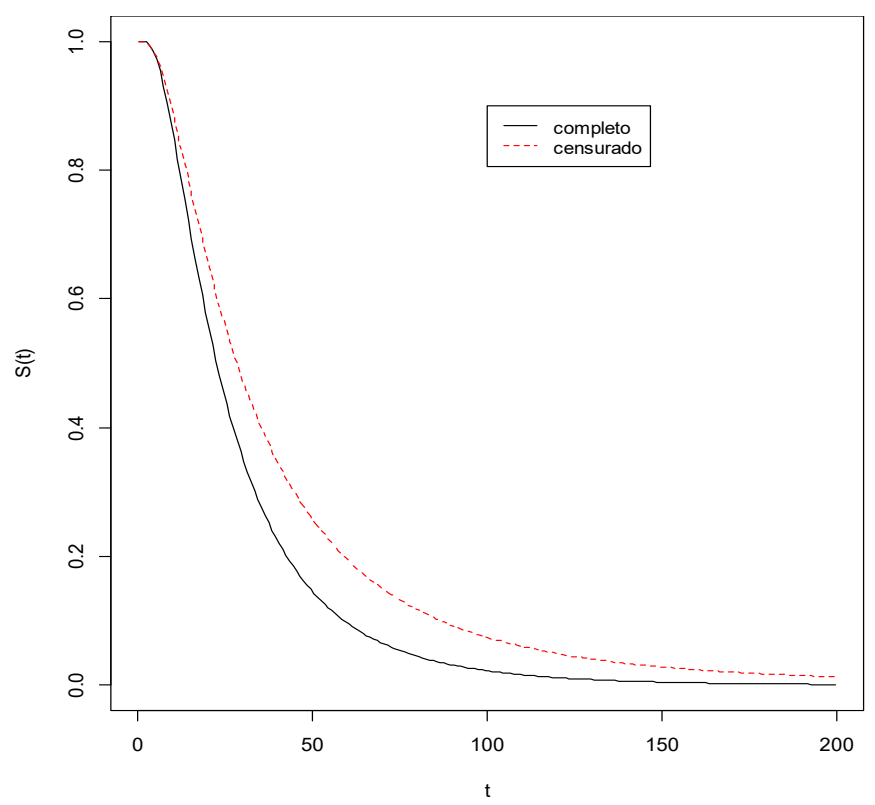

Figura 8. Função de sobrevivência estimada considerando os dados completos.

Analisando os gráficos da função de sobrevivência correspondentes aos dados censurados e não-censurados na Fig. 9 pode-se observar que a probabilidade de sobrevivência é superior sob dados censurados em relação aos dados nãocensurados até pouco mais de 200 horas. A partir desse momento, os dados não-censurados passam a acarretar uma probabilidade de sobrevivência superior aos dados censurados.

\section{CONCLUSÕES}

Neste artigo uma análise Bayesiana foi desenvolvida com o auxílio de métodos de simulação MCMC (Monte Carlo via cadeias de Markov) para avaliarmos a performance das prioris propostas na presença de dados censurados sob a distribuição Birbaum-Saunders. Mostramos que o uso de funções cópula na construção de distribuições a priori pode ser um método alternativo e de grande interesse prático na inferência Bayesiana. 


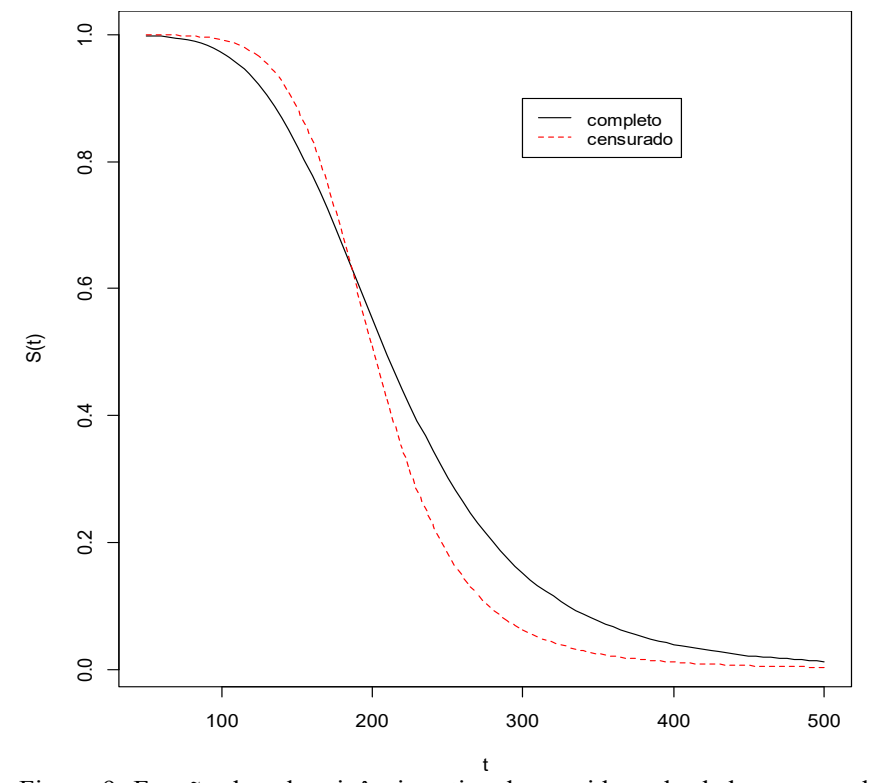

Figura 9. Função de sobrevivência estimada considerando dados censurados tipo II e completos.

Através de cópulas é possível acrescentarmos informações sobre a dependência entre os parâmetros.

Os resultados obtidos através dos sumários a posteriori e intervalos de credibilidade para ambas prioris são similares na ausência de informação sob censura tipo II.

Através de dois exemplos aplicados verificamos os efeitos causados na estimação ao não considerarmos na análise os dados censurados presentes em um experimento. A utilização de dados censurados proporcionará uma estimação mais precisa e confiável.

\section{REFERÊNCIAS}

[1] Z.W Birnbaum, S.C. Saunders, A new family of life distribution, Journal of Applied Probability, 6, 319-327, 1969.

[2] Z.W Birnbaum, S.C. Saunders, Estimation for a family of life distribution with applications to fatigue, Journal of Applied Probability, 6, 328-347, 1969.

[3] J.R. Rieck, Statistical Analysis for the Birnbaum-Saunders fatigue life distribution, Unpublished $\mathrm{PhD}$ thesis, Clemson University, Dept of Mathematical Sciences, 1989.

[4] J. Lawless, Statistical models and methods for lifetime data, John Wiley, New York, 1982.

[5] R.B. Nelsen, An introduction to copulas, Springer-Verlag, New York, 1999.

[6] P.K. Trived, D.M. Zimmer, Copula Modelling, Now Publishers, 2005.

[7] P.K. Trived, D.M. Zimmer, Copula Modelling: an introduction to practiciones, Form dations and trends in econometrics, 1, 1, 1-111, 2005.

[8] D. Morgenstern, Einfache Beispiele Zweidimensionaler Verteilungen, Mitteilingsblatt fur Matematische Statistik, 8, 234-253, 1956.

[9] G. Casella, E.I. George, Explaining the Gibbs sampler, The American Statistician, 46, 3, 167, 174, 1992.

[10] S. Chib, E. Greenberg, Understanding the Metropolis-Hastings algorithm, The American Statistician, 49, 327 - 335, 1995.

[11] J.I. McCool, Inferential techniques for Weibull populations. Aerospace Research Laboratories Report ARL TR74-0180. Wright Patterson Air Force Base, Dayton, OH, 1974.

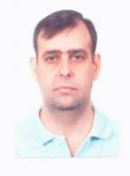

Fernando Antonio Moala, possui graduação em Matemática (Bacharelado) pela UNESP (1988), S. J. do Rio Preto, mestrado em Ciências da Computação e Matemática Computacional (ênfase em Estatística) pela USP (1993), São Carlos, e $\mathrm{PhD}$ em Estatística na Universidade de Sheffield (2006), Inglaterra. Em 2014-2015 realizou um ano de PósDoutorado na McMaster University, Canadá. Atualmente é professor assistente doutor da UNESP, campus de Presidente Prudente. Tem experiência na área de Probabilidade e Estatística, com ênfase em Inferência Bayesiana e Análise de Confiabilidade, atuando principalmente nos seguintes temas: elicitação de prioris, prioris não-informativas, estimação de modelos e análise de confiabilidade.

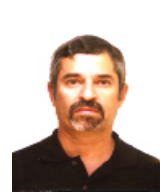

Jorge Alberto Achcar, possui graduação em matemática pela UNESP em 1971, mestrado em estatística pelo IME-USP, São Paulo em 1976, doutorado (PhD) em estatística pela Universidade de Wisconsin-Madison, Estados Unidos em 1982. Professor livre-docente pelo ICMC-USP em 1990.Possui pos-doutorado pelas Universidades de Nottingham,Inglaterra; de Connecticut, EUA e CarnegieMellon, EUA. Professor visitante em diversas universidades brasileiras e estrangeiras. Atua como docente na UNESP, UFSCAR e USP desde 1974 na área de estatística. Atualmente atua como professor colaborador no departamento de medicina social da faculdade de medicina da USP, Ribeirão Preto. Tem atuado em docência e pesquisa em diversas áreas de estatística, com ênfase em inferência Bayesiana, estatística aplicada a medicina, a engenharia e a economia, analise de sobrevivência e confiabilidade, probabilidade, planejamento de experimentos, inferência estatística, análise multivariada e processos estocásticos aplicados.

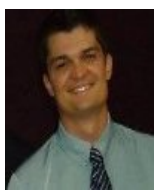

Robson Pedreiro Gimenez, possui graduação em Estatística (Bacharelado) pela UNESP (2013), Presidente Prudente. Atualmente é mestrando em Economia pelo INSPER. Trabalhou como consultor estatístico nas empresas Intrabase e FEA (USP). Atualmente é Analista de Modelagem no Banco Bradesco S/A. 